\title{
Optimization of an aperiodic sequential inspection and condition-based maintenance policy driven by Value of Information
}

\author{
William Fauriat ${ }^{\mathrm{a}, *}$, Enrico Zio ${ }^{\mathrm{b}, \mathrm{c}}$ \\ ${ }^{a}$ Laboratoire de Génie Industriel, CentraleSupélec, Université Paris-Saclay, Gif-sur-Yvette, France \\ ${ }^{b}$ Center for Research on Risk and Crisis (CRC), Mines ParisTech, PSL University, Sophia Antipolis, France \\ ${ }^{c}$ Energy Department, Politecnico Di Milano, Milan, Italy
}

\begin{abstract}
The issue of the optimal planning of inspection and maintenance actions for a randomly deteriorating system constitutes a difficult sequential decision-making problem in which the objective is generally to achieve minimal life-cycle cost. For mathematical tractability, most approaches rely either on the consideration of specific maintenance strategies, e.g. Periodic Inspection and Replacement (PIR), whose defining parameters are optimized, or on time-and-space-state discretization using Markov Decision Process (MDP) models and resolution through policy iteration. In both cases, optimality may be hard to guarantee. In this paper, the decision-theoretic concept of Value of Information (VoI) is used as a metric to guide resource prioritization in time, that is, to schedule inspections in a piecewise optimal manner.

An aperiodic sequential inspection policy is proposed, where the determination of the next best time for inspection, or replacement, is based on the current condition and on the computed expected gain from possible inspections, i.e. on a VoI metric. This policy can be implemented when the current condition is known from imperfect inspection or processing of condition-monitoring data. Also, more generally, a discussion is proposed on the use of VoI as a guide for information collection in life-cycle management.
\end{abstract}

Keywords: Value of Information, Condition-Based Maintenance, inspection planning, maintenance optimization, sequential decision-making, renewal theory, imperfect information

\begin{tabular}{cc} 
Acronyms & \\
\hline VoI & Value of Information \\
CBM & Condition-Based Maintenance \\
PIR & Periodic Inspection and Replacement \\
BR & Block Replacement \\
MDP & Markov Decision Process \\
PHM & Prognostic and Health Management \\
\hline
\end{tabular}

\begin{tabular}{cc} 
Notations & \\
\hline$X(t)$ & Degradation process \\
$F(t)$ & Failure probability function \\
$F_{\mid z}(t)$ & Conditional failure probability function \\
$z(\tau)$ & Condition (degradation) of component \\
$L(s, a)$ & Loss outcome for action $a$ in state $s$ \\
$C_{\infty}(a)$ & Unconditional average cost per unit time \\
& for replacement at time $a$ \\
$C_{\infty \mid z}(a)$ & Conditional average cost \\
$\operatorname{VoI}(\tau)$ & VoI for inspection at $\tau$ \\
\hline
\end{tabular}

\footnotetext{
* Corresponding Author

Email addresses: william.fauriat@centralesupelec.fr (William Fauriat), enrico.zio@polimi.it (Enrico Zio)
}

\section{Introduction}

Systems, structures or components that are subject to random degradation often require maintenance. Preventive actions such as inspections, repairs or replacements 5 can be carried out with the objectives to limit the likelihood of failure, thereby improving reliability and safety, minimize downtime and associated costs, extend life, etc. In many practical cases, the issue of maintenance optimization or planning, essentially consists in determining 10 what actions are necessary and when to apply them, in view of achieving minimal life-cycle cost. In mathematical terms, this issue constitutes a sequential decision-making problem in an uncertain environment and its resolution is a difficult challenge.

To address such challenge, it is generally necessary to use stochastic models in order to describe and quantify reliability, random degradation processes or the outcome of maintenance decisions, see e.g. early works of [1] and [2]. In this framework, much research effort has been dedicated 20 to finding 'optimal' inspection and maintenance strategies, especially in the structural and reliability engineering community, see e.g. [3, 4, 5, 6]. The formulation of the problem and the suitability of different resolution approaches may vary depending on the considered application. The selec25 tion of the most cost-efficient strategy is seldom straight- 
forward. In this paper, we restrict our attention to preventive maintenance and single component analysis only. Good reviews on the extensive literature on maintenance 85 may be found in $[7,8,8,9,10]$.

A maintenance strategy or policy can be determined by actions that are carried out at specific time instances and by actions that depend on available information on the current condition of the component. They are respectively 90 denoted as time(or age)-based maintenance and conditionbased maintenance (CBM) strategies. Roughly speaking, the approach used for the resolution of the maintenance optimization problem, or equivalently, for the selection of a preventive maintenance policy, belongs to one of the two 95 following categories.

In the first category, one may consider policies with specific structures, i.e. set of rules depending on time or condition, that are parametrized by one or several design values, such as the inspection interval or the preventive replacement limit, see examples in $4,6,11,12,13,14,15$, 16, 17, 18]. Those parameters are then optimized, given information on the degradation process, in order to reach ${ }^{100}$ a desired objective, e.g. minimal average life-cycle cost. Quantitative analysis of the random outcome of such policies often relies on stochastic models with various degrees of complexity and on the use of renewal theory [1]. This is the type of method considered in this paper.

In the second category, the sequential decision problem is explicitly formulated, the degradation and decision processes are discretised and represented by Markov Decision

${ }_{55}$ Process (MDP) models. In this case, the policy, i.e. the mapping between known state and actions, is computed through adapted resolutions scheme, see seminal work of [19, 20], and 21] for details on numerical resolution. 110

Independently of the resolution method, it is gener-

60 ally recognized that collecting additional information on the uncertain state of a component, through inspection or exploitation of condition-monitoring data, can lead to better outcomes, as far as subsequent maintenance decisions are concerned. In this paper, it is proposed to make 65 use of the concept of Value of Information (VoI) to quantify such improvements and help plan information collection in a rational manner. This concept was developed in the field of statistical decision theory, in the seminal ${ }_{115}$ works of [22, 23, 24], and has received increasing atten-

70 tion recently in the context of structural health monitoring [25, 26, 27, 28, 29, 30], risk-based inspection 31, 32, 33] and maintenance planning 34, 35, 36, 37, 38.

Essentially, VoI quantifies the expected gain, in terms of decisions outcome, that may be achieved with the col75 lection of an additional piece of information. In this paper, it is shown that VoI can be a relevant metric to help decide whether or not and when to collect condition information. As this operation may come at cost, VoI is ${ }^{120}$ used as a resource prioritization metric in the time do-

so main. It helps guiding the resolution of the maintenance optimization problem. More specifically, the VoI metric is employed to construct an aperiodic sequential inspection policy, where inspections are sequentially scheduled in a piecewise optimal manner.

The paper is organized as follows. In Section 2, the issue of maintenance optimization is addressed in the context of continuous-time degradation and some elements are given on Gamma process modeling and renewal theory. The notion of Value of Information (VoI) is defined and discussed in Section 3. The computation of VoI within the previously defined framework is detailed in Section 4. With the VoI metric, an aperiodic sequential inspection policy is proposed and experimentally tested in Section 5 . The use of VoI for resource prioritization and information collection is discussed in Section 6. Conclusions are given in Section 7

\section{Continuous-time degradation and maintenance optimization}

\subsection{Gamma process for degradation modeling}

Gamma processes are often used to represent continuoustime degradations, as they are strictly non-decreasing and quite amenable to mathematical treatment. For an extensive review on the use of gamma processes for maintenance modeling and optimization, see [10]. A stochastic process $X(t)$ is a gamma process if it has gamma distributed independent increments

$$
X(t)-X(s) \sim \mathrm{Ga}(v(t)-v(s), u)
$$

where $\mathrm{Ga}: x \rightarrow \mathrm{Ga}(x, v, u)$ is the gamma probability density function, with shape parameter $v$ and scale parameter $u$. At any time, the expectation and variance of the degradation process are:

$$
\begin{gathered}
E[X(t)]=v(t) / u \\
\operatorname{Var}[X(t)]=v(t) / u^{2}
\end{gathered}
$$

The trend or mean function $v: t \rightarrow v(t)$, can be any continuous non-decreasing function, with $v(0)=0$. In this paper, we consider only trends of the form $v: t \rightarrow \lambda t^{b}$ with $\lambda>0$ and $b=1$, that is, stationary gamma processes, for ease of computation, with no loss in generality for the proposed maintenance policy.

If it is assumed that the component fails when the degradation reaches a predetermined level $y$, then the failure probability at time $t$, can be obtained, see [10], using:

$$
\begin{gathered}
F(t)=\operatorname{Pr}\left[T_{y} \leq t\right]=\operatorname{Pr}[X(t) \geq y] \\
=\int_{x=y}^{\infty} f_{X(t)}(x) d x=\frac{\Gamma(v(t), y u)}{\Gamma(v(t))}
\end{gathered}
$$

where $\Gamma(a, x)=\int_{z=x}^{\infty} z^{a-1} e^{-z} d z$ is the incomplete gamma function for all $a>0$ and $x>0$, and $\Gamma: x \rightarrow \Gamma(x)$ is the traditional gamma function. 


\subsection{Maintenance modeling and optimization}

With random degradation, the outcome of a maintenance policy is stochastic. The purpose of maintenance optimization is to plan preventive actions in order to get the best possible outcome according to some selected cri- ${ }^{175}$ teria, usually expected cost. Here the following actions are considered: inspection at $\operatorname{cost} c_{i}$, preventive replacement at cost $c_{r}$ and corrective replacement, upon failure of the component, at cost $c_{c}$, where generally $c_{c}>>c_{r}>>c_{i}$.

In practice, many different situations may have to be described, e.g. perfect or imperfect inspections, perfect or imperfect repairs, instantaneous or non-instantaneous tenance crew may or may not be readily available. The objective here is not to cover all possible applications and the ideas presented hereinafter, on a rather simple but sufficiently representative case, could apply to more complex ${ }_{185}$ practical cases, at the expense of more effort in domain specific modeling.

The average outcome of the maintenance policy, on an infinite time horizon, can be more easily computed in the case of renewal cycles, that is, cycles or times histories that ${ }_{190}$ bring a component back to its original condition. Here, this is the case for both preventive replacement or corrective replacement upon failure, whichever occurs first. A relevant metric to consider in this context is the average cost per unit time $C_{\infty}$. According to renewal theory, see e.g. 39, 1, 9], the latter can be computed using:

$$
C_{\infty}=\lim _{t \rightarrow \infty} \frac{C(t)}{t}=\frac{E[C(S)]}{E[S]}
$$

where $C(t)$ is the accumulated maintenance cost at time $t, \mathrm{E}[\mathrm{C}(\mathrm{S})]$ is the average total maintenance cost of renewal cycles, $E[S]$ is the average length of renewal cycles.

Let us consider a policy, a so called time(or age)-based ${ }^{200}$ replacement policy, in which the decision variable $a$ is the date at which the component is supposed to be preventively replaced. In this case, the cost per unit time may be computed by averaging over all possible outcomes of the policy, namely preventive replacements and (instantaneous) corrective replacements upon (self-revealing) failure

$$
C_{\infty}(a)=\frac{c_{c} F(a)+c_{r}(1-F(a))}{\left(\int_{0}^{a} u d F(u)\right) F(a)+a(1-F(a))}
$$

where $F($.$) is the failure probability function in (4). F(a)$ components will be failed before time $a$ with $\operatorname{cost} c_{c}$ and average life $\left(\int_{0}^{a} u d F(u)\right)$ and $1-F(a)$ components will life $a$. Using (6), one has a direct relationship between ${ }^{210}$ the selected policy, i.e. the decision variable $a$, and the expected outcome $C_{\infty}(a)$.

Let us note that, here, the use of renewal theory allows

maintenance planning, as for this simple policy, the objective is only to find $a^{*}=\arg \min _{a} C_{\infty}(a)$. Roughly said, by adjusting $a$ one seeks an optimal policy, as waiting too little will lead to replacements that are too frequent, while waiting too long will produce an excessively large number of failures over time.

\section{Value of Information}

\subsection{Definition of $V_{o I}$}

As described in 25], "the value of a piece of information depends on its ability to guide our decision". Value of Information ( VoI) is a concept rooted in Bayesian theory and decision theory that provides a rational approach to attach a value to a piece of information. Formally, see [22], VoI is calculated as the difference in expected cost (or loss) between the outcome of the optimal decisions that may be taken with and without the collection of additional information.

If $L(s, a)$ is the loss that is incurred when the component is in state $s$ and the decision-maker selects action $a$, then VoI can be calculated according to:

$$
\mathrm{VoI}=L^{*}(\emptyset)-E_{Z}\left[L^{*}(z)\right]
$$

where $L^{*}(\emptyset)$ represents the minimal expected loss (i.e. associated to the optimal decision $a^{*}$ ) when no additional information is available and $L^{*}(z)$ represents the minimal expected loss when the information $z$ has been observed, thus 'modifying' the knowledge on the uncertainty associated to the component's state to the posterior $s \mid z$. As one does not know beforehand what the observation is going to be, $L^{*}(z)$ has to be averaged over all possible observations. Hence, this approach is often called pre-posterior analysis 22].

Giving the expression of the minimal expected loss and making the dependence on uncertainty explicit, (7) becomes:

$$
\begin{aligned}
\mathrm{VoI}=\min _{a \in A} \int & L(s, a) p(s) d s \\
& -\int\left(\min _{a \in A} \int L(s, a) p(s \mid z) d s\right) p(z) d z
\end{aligned}
$$

where $A$ is the set of all possible actions, $p(s)$ is the prior distribution over the component's state and $p(s \mid z)$ is the posterior distribution given an observation $z$.

Roughly speaking, there is value in collecting information $z$ if knowing that the uncertainty is then reduced from $p(s)$ to $p(s \mid z)$ allows us to make decisions which outcome $L^{*}(z)$ is 'better' on average than the optimal outcome $L^{*}(\emptyset)$ that is obtained from an unconditional decision based on the initial uncertainty on the component's state $p(s)$. 


\subsection{VoI analysis and use}

In general, the computed VoI has to be compared with the cost of collecting the considered information. Then, the collection is worth realizing only if its cost does not exceed the additional value it can generate on average, namely VoI. This metric provides a rational approach to compare inspection devices, mechanisms or procedures that provide different pieces of information on the component's state and with different levels of precision.

The expression in (8) describes the general framework for the computation of VoI. For each practical application, the interest in collecting information according to a collection (or observation) mechanism specified by the probability measure $p(s \mid z)$, will depend on the decision context via $L(s, a)$ and $p(s)$, which also have to be specified.

From a practical perspective, carrying out a VoI analysis thus consists in two operations: first, specify the decision context and observation or uncertainty reduction mechanism and second, compute both the conditional and unconditional estimations and optimizations in (8). In this paper, the decision context is specified via $C_{\infty}(a)$ in (6) which may be written as:

$$
C_{\infty}(a) \Leftrightarrow \int L(s, a) p(s) d s
$$

\section{VoI metric for inspection time determination}

\subsection{Inspection and conditional replacement}

Let us consider the maintenance actions proposed in Section 2.2, namely preventive replacement at cost $c_{r}$, corrective replacement at $\operatorname{cost} c_{c}$ and inspection at cost $c_{i}$. If an inspection is made at time $\tau$ and the condition of the 260 component is noted as $z(\tau)$ then, provided the component is not failed, i.e, $z(\tau)<y$, the conditional average cost per unit time $C_{\infty \mid z}$ can be computed using (6), adding the cost of one inspection and substituting $F(t)$ in (4) by:

$$
F_{\mid z}(t)=\frac{\Gamma(v(t-\tau),(y-z(\tau)) u)}{\Gamma(v(t-\tau))}
$$

for all $t>\tau$ and with $v(t)=v t, v>0$. Indeed, when $z(\tau)$ is known, failure occurs when the 'remaining capacity' of the component is consumed, that is, when degradation is increased by $y-z(\tau)$ units, with an increase in mean value $\Delta v=v \Delta t$. This gives the following expression for the cost per unit time, conditional on the observation at time $\tau$

$$
C_{\infty \mid z(\tau)}(a)=\frac{c_{c} F_{\mid z}(a)+c_{r}\left(1-F_{\mid z}(a)\right)+c_{i}}{\left(\int_{\tau}^{a} u d F_{\mid z}(u)\right) F_{\mid z}(a)+a\left(1-F_{\mid z}(a)\right)}
$$

for all $t>\tau$ and $z(\tau)<y$.

The unconditional and conditional costs per unit time functions are displayed as an example in Figure 1, with an inspection at time $\tau=100$ hours, two possible outcomes $z_{1}=30$ and $z_{2}=70$, and for the parameters given in ${ }^{275}$ Table 1 .

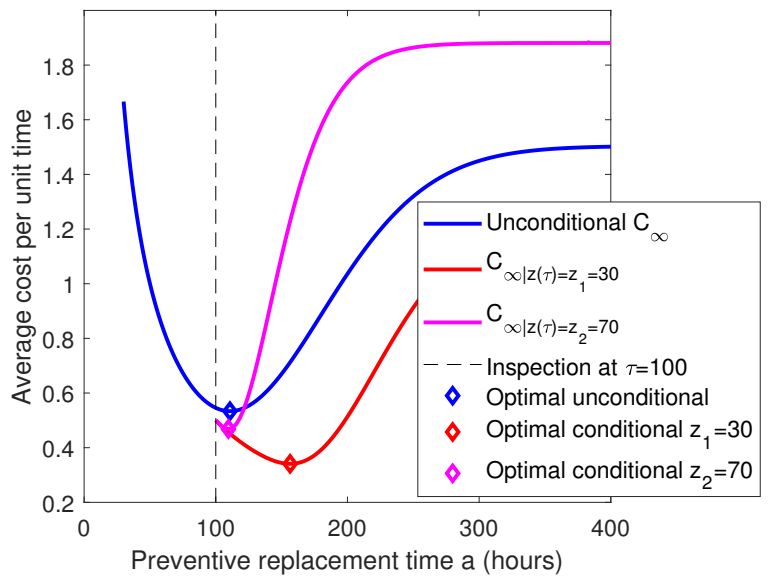

Figure 1: Unconditional and conditional cost per unit time functions.

\begin{tabular}{ccc} 
Parameter & Notation & Value \\
\hline Failure threshold & $y$ & 100 \\
Power law exponent & $b$ & 1 \\
Degradation variability & $u$ & 8 \\
Degradation rate & $v$ & $y /\left(200^{b} u\right)$ \\
Corrective replacement cost & $c_{c}$ & 300 \\
Preventive replacement cost & $c_{p}$ & 50 \\
Inspection cost & $c_{i}$ & 1
\end{tabular}

Table 1: Parameters of the considered decision context. Degradation rate is fixed for failure on average at time $t=200$ hours for simplicity of visualization and analysis.

It is seen in Figure 1, that the optimal decision is conditional on the outcome of the inspection. In this example, when the degradation has progressed more slowly up to time $\tau, z_{1}<z_{2}$, it is better, on average, to wait longer before proceeding to preventive replacement with :

$$
\underset{a}{\arg \min } C_{\infty \mid z_{1}}(a)>\underset{a}{\arg \min } C_{\infty \mid z_{2}}(a)
$$

\subsection{Calculation of VoI for a single inspection}

With the decision context defined in section 4.1 and with (perfect) inspection taken as the uncertainty reduction mechanism, VoI can be computed using (8) and (9), which becomes here:

$$
\operatorname{VoI}(\tau)=\min _{a} C_{\infty}(a)-E_{z(\tau)}\left[\min _{a} C_{\infty \mid z(\tau)}(a)\right]
$$

where the unconditional $C_{\infty}(a)$ and conditional $C_{\infty \mid z(\tau)}(a)$ costs are already expected values, namely on the probability measures that have been denoted as $p(s)$ and $p(s \mid z)$ in (8). Here, for the distributions $p(s)$ and $p(s \mid z), s$ is to be understood as the 'resolution of uncertainty' on the time interval $[0, a]$, that is, a preventive replacement or a corrective replacement, when the action is originally planned at time $a$. The representation of the randomness measured by $p(s)$ and $p(s \mid z)$ is associated to the gamma degradation 
process model, through the use of $F(a)=\operatorname{Pr}(s[0, a]<y)$ and $\left.F\right|_{z}(a)=\operatorname{Pr}(s[\tau, a]<y \mid z(\tau)=z)$.

$C_{\infty}(a)$ and $C_{\infty \mid z(\tau)}(a)$ can be computed using the closedform expressions in (6) and (11). The unconditional and conditional optimal decisions:

$$
\begin{gathered}
a^{*}=\underset{a}{\arg \min } C_{\infty}(a) \\
a_{\mid z}^{*}=\underset{a}{\arg \min } C_{\infty \mid z(\tau)}(a)
\end{gathered}
$$

can be obtained via numerical optimization schemes.

As mentioned in Section 3.1, the outcome of the observation step $z(\tau)$ cannot be known in advance. Thus, the expected gain has to be averaged over the probability measure $p(z(\tau))$. With the choice of a gamma degradation process, one has:

$$
z(\tau) \sim \operatorname{Ga}(v(\tau), u) .
$$

Also, for values $z(\tau)>y$, failure has occurred and it is no longer possible to act to prevent the latter. In such case, us use the following average cost value $C_{\infty \mid z(\tau)>y}={ }_{325}$ $c_{c} / \tau$ (which is an optimistic estimate as failure may have occurred prior to $\tau$ ). In practice, the quantity:

$$
E_{z(\tau)}\left[\min _{a} C_{\infty \mid z(\tau)}(a)\right]
$$

is computed using numerical integration and an inner loop of numerical optimization.

One readily sees that the VoI computed in (13) depends on the inspection time $\tau$. This inspection time practically defines the uncertainty reduction or observa- ${ }_{335}$ tion mechanism that is considered. Then, the objective is to use $\operatorname{VoI}(\tau)$ as a resource prioritization metric and find most relevant time for inspection, as far as average cost is concerned. The evolution of VoI as a function of the inspection time $\tau$ is given in Figure 2 for the parameters in Table 1. The best value for (the first) inspection is around $\tau \approx 97$ hours. Inspecting earlier or later is less profitable.

Generally, VoI is not negative, according to the principle that "more information cannot hurt". Nonetheless, the choice here is to consider negative VoIs, or more precisely, allow the possibility of net loss of value. Let us note that the VoI calculated using (11) and (13) already includes the cost of inspection $c_{i}$. Thus, it is in fact a net gain or loss of value, corresponding to the expected outcome once the inspection has been effectively performed. For consistency of discussion, the metric used to propose a conditional inspection policy in this paper will be described as a VoI metric regardless.

In Figure 2, negative VoIs occur both for small values of $\tau$ and for high values of $\tau$. For early inspections, there is no particular improvement on the average outcome of conditional decisions but cost $c_{i}$ is payed regardless, which translate to a net loss. For late inspections, the proportion of failed components increases when, in theory, the best

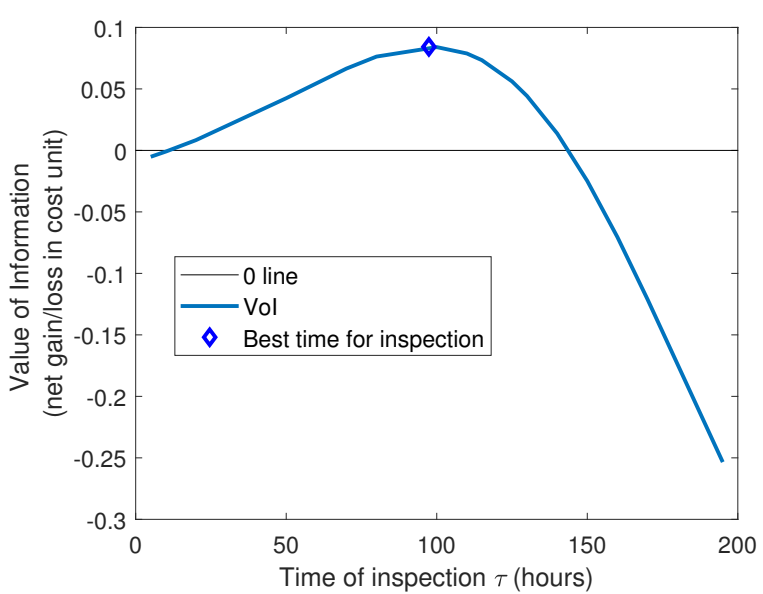

Figure 2: Value of Information (here expressed as a net gain/loss in terms of cost units) as a function of the inspection time $\tau$.

decision in the sense of (8) is not to wait until failure and proceed to a preventive replacement at an optimal time which is then prior to $\tau$. Consequently waiting too long for inspections, before effectively acting, yields nonoptimal decisions, driving net loss up. From the theoretical perspective, the collection of $z$ may restrain the action space from $A$ to $A^{\prime}$ (where preventive replacement is no longer an option), which is not the standard framework for VoI computation in (8) and explains in part why we get negative VoIs here.

To clarify, let us simply consider that if $\mathrm{VoI}$ is negative at time $\tau$ (no matter the magnitude or its exact interpretation), it is not worth inspecting at time $\tau$ since that decision would correspond to a net loss on average.

\subsection{Calculation of VoI for subsequent inspections}

With the use of conditional gamma failure probability functions, it is possible to calculate easily the VoI corresponding to the difference between the unconditional cost $C_{\infty \mid z_{1}, \tau_{1}}(a)$, starting from the outcome of the first inspection yielding $\left(z_{1}, \tau_{1}\right)$, and the conditional cost for a subsequent inspection $C_{\infty \mid z\left(\tau_{2}\right), z_{1}, \tau_{1}}(a)$. Simply said, a new problem, translated by $\left(z_{1}, \tau_{1}\right)$, is considered after the first inspection, i.e. on a new time window, where a new VoI can be computed. For this translated problem, the unconditional and costs are:

$$
\begin{array}{r}
C_{\infty \mid z_{1}, \tau_{1}}(a)= \\
\frac{c_{c} F^{\prime}(a)+c_{r}\left(1-F^{\prime}(a)\right)+c_{i}}{\left(\int_{\tau_{1}}^{a} u d F^{\prime}(u)\right) F^{\prime}(a)+a\left(1-F^{\prime}(a)\right)+\tau_{1}}
\end{array}
$$

with:

$$
F^{\prime}(t)=\frac{\Gamma\left(v\left(t-\tau_{1}\right),\left(y-z_{1}\right) u\right)}{\Gamma\left(v\left(t-\tau_{1}\right)\right)}
$$

for $t>\tau_{1}, z_{1}<y$ and: 


$$
\begin{aligned}
& C_{\infty \mid z\left(\tau_{2}\right), z_{1}, \tau_{1}}(a)= \\
& \frac{c_{c} F_{\mid z}^{\prime}(a)+c_{r}\left(1-F_{\mid z}^{\prime}(a)\right)+2 c_{i}}{\left(\int_{\tau_{2}}^{a} u d F_{\mid z}^{\prime}(u)\right) F_{\mid z}^{\prime}(a)+a\left(1-F_{\mid z}^{\prime}(a)\right)+\tau_{1}}
\end{aligned}
$$

with:

$$
F_{\mid z}^{\prime}(t)=\frac{\Gamma\left(v\left(t-\tau_{2}\right),\left(y-z_{1}-z\left(\tau_{2}\right) u\right)\right.}{\Gamma\left(v\left(t-\tau_{2}\right)\right)}
$$

for $t>\tau_{2}, z_{1}+z\left(\tau_{2}\right)<y$.

The expression for the computation of VoI, for a problem starting in $\left(z_{1}, \tau_{1}\right)$ is:

$$
\begin{aligned}
& \operatorname{VoI}_{z_{1}, \tau_{1}}\left(\tau_{2}\right)= \\
& \quad \min _{a} C_{\infty \mid z_{1}, \tau_{1}}(a)-E_{z\left(\tau_{2}\right)}\left[\min _{a} C_{\infty \mid z\left(\tau_{2}\right), z_{1}, \tau_{1}}(a)\right]
\end{aligned}
$$

It can be used for any i-th inspection, provided the component has lived up to $\left(z_{i-1}, \tau_{i-1}\right)$, which is no longer random from that point.

An example of a second rank inspection is given in Figure 3, after a first inspection at optimal time $\tau_{1} \approx^{385}$ 97hours.

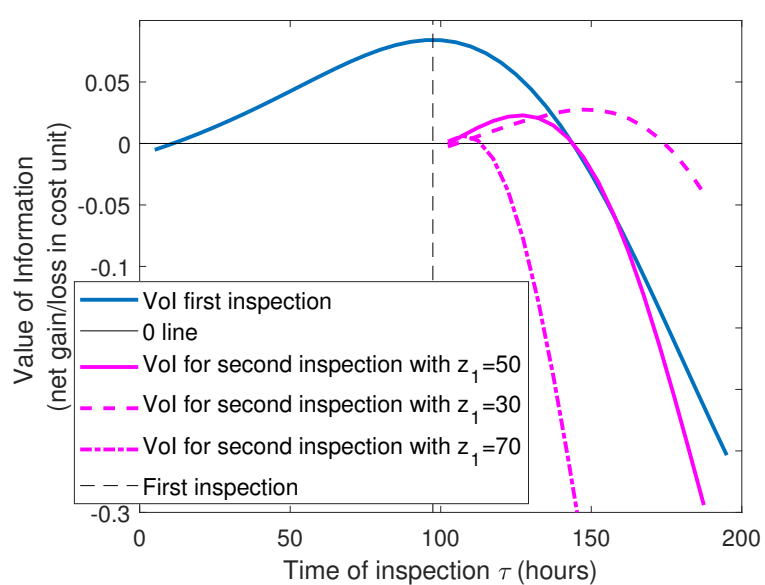

Figure 3: Value of Information for first and second inspections. In the latter case, three outcomes of the first inspection are considered.

Depending on the outcome of the first inspection at $\tau_{1}$, it may or may not be relevant to inspect again, and the ${ }_{405}$ best time for next inspection is dependent on the current condition of the component at the point where a new VoI is computed.

\subsection{VoI computation with imperfect inspection}

The information obtained from inspection or from the processing of condition-monitoring data may not be perfect. This has to be taken into account when making decisions and estimating VoI. Let us assume that the true
370 condition $z$ of the component, unknown, is normally distributed around the value obtained from inspection $z_{i}$ :

$$
z \sim N\left(z_{i}, \sigma_{i}\right)
$$

This type of error model is quite classical for inspection or measurement uncertainty and may apply equally well for the error committed by an imperfect estimation model in the case of the of processing condition-monitoring data. The degree of precision of the inspection can be controlled via the noise level or inspection dispersion $\sigma_{i}$.

The solution which is proposed here in order to make a decision that accounts for inspection uncertainty is the following. The conditional cost function is averaged over the Gaussian distribution and the best decision is calculated according to:

$$
\begin{aligned}
a^{*}=\underset{a}{\arg \min } C_{\infty \mid z_{i}}^{u}(a) & = \\
& \underset{a}{\arg \min } E_{z \sim N\left(z_{i}, \sigma_{i}\right)}\left[C_{\infty \mid z}\right](a)
\end{aligned}
$$

where $E_{z \sim N\left(z_{i}, \sigma_{i}\right)}[$.$] designates the average taken over the$ Gaussian distribution, centered on the inspection $z_{i}$. The integration is done numerically for different values $a$, to build the numerical function :

$$
C_{\infty \mid z_{i}}^{u}(a)=E_{z \sim N\left(z_{i}, \sigma_{i}\right)}\left[C_{\infty \mid z}\right](a)
$$

Then, an optimization scheme can be applied to find the 'optimal' decision. The latter is described as 'optimal' since, on average, i.e. no matter what the true condition, yet unknown, ends up being, the selected $a^{*}$ leads to the minimal cost.

The averaged conditional cost function $C_{\infty \mid z_{i}}^{u}(a)$ is visible in Figure 4. The inspection uncertainty is selected quite large for clear visualization. It is seen that when uncertainty is taken into account (averaged conditional cost), it is better to proceed to preventive replacement slightly earlier, as one may have underestimated degradation in some instances (due to measurement noise).

VoI can then be computed using (13) and substituting the standard conditional cost function $C_{\infty \mid z}(a)$ by the averaged conditional cost function $C_{\infty \mid z_{i}}^{u}(a)$ defined in (25). The integration in (13) is done assuming that the prior distribution for observation is $p\left(z_{i}\right)=p(z)$, where $z \sim$ $\mathrm{Ga}(v \tau, u)$. Let us note that this assumption is reasonable if $\sigma_{i}$ is not too important.

In Figure 5. VoIs are computed for different intensity of the inspection uncertainty $\sigma_{i}$. It is seen that the maximum value of $\mathrm{VoI}$ decreases when there is uncertainty in the inspection. Simply put, an imperfect inspection reduces uncertainty less, in the sense of (8), and the improvement in terms of decision outcome is not as profitable. 


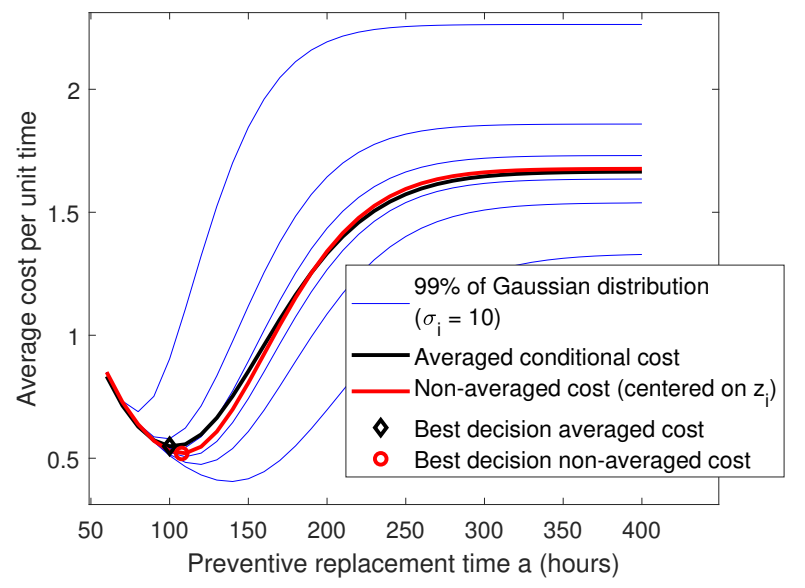

Figure 4: Averaging of conditional cost functions. Blue solid curves correspond to different realizations of $z \sim N\left(z_{i}, \sigma_{i}\right)$.

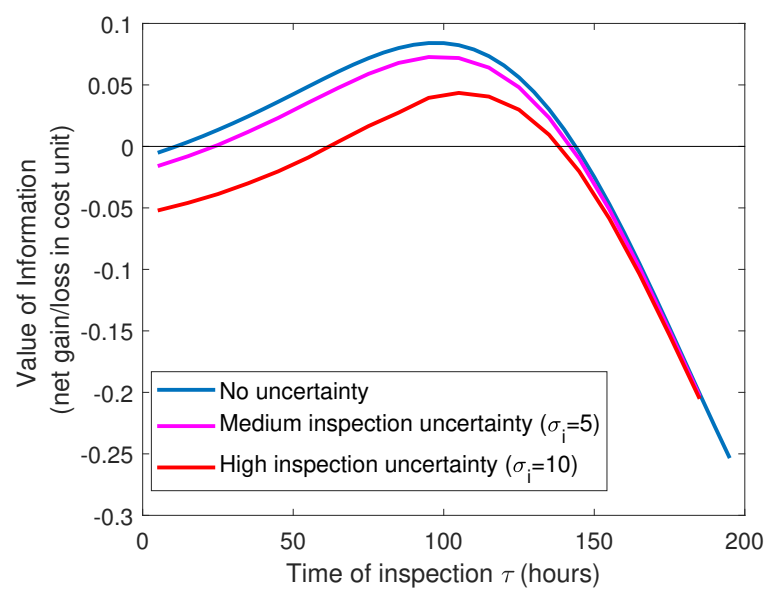

Figure 5: Effect of inspection uncertainty on VoI.

\section{Proposed sequential inspection policy}

\subsection{Policy definition and illustration}

With the elements presented above, the calculation of 415 VoI can be performed, for an inspection and preventive replacement context, using (13) for the first inspection and (22) for any following i-th inspection conditional on the observation made at the previous step.

Based on this, the following sequential inspection policy is proposed. It consists in computing, at each step, the VoI as a function of the inspection time $\tau_{i}$ and observed condition $z\left(\tau_{i}\right)$, and selecting the next best time for inspection as the time when VoI is maximum, provided the latter in positive. This is repeated until the maximum of $\mathrm{VoI}$ becomes negative and it is no longer profitable to inspect. When this happens, the component is preventively replaced at the optimal time conditioned on the last inspection. The details of the implementation of the sequential policy are given in Algorithm 1 .

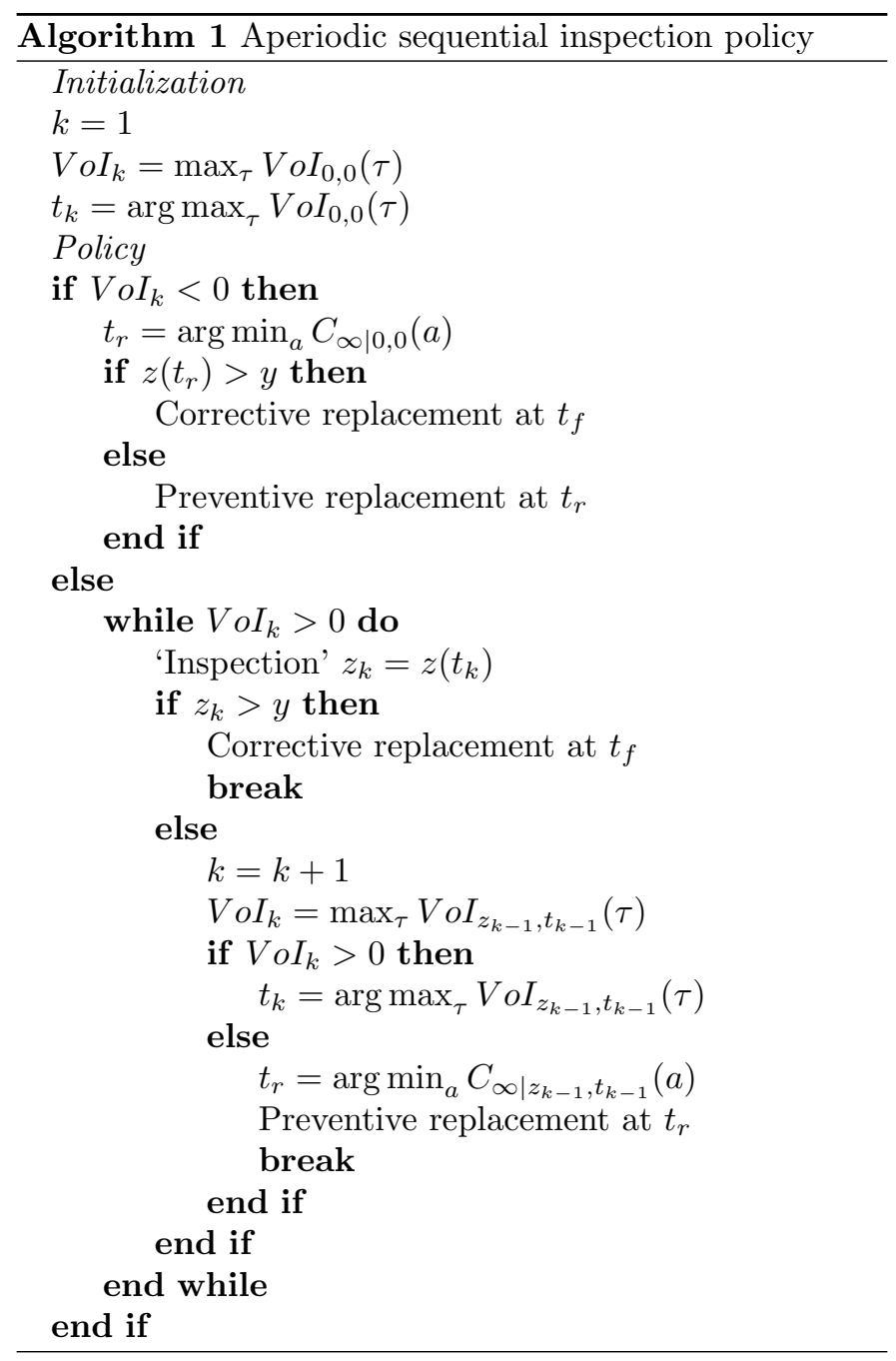

As an application of the proposed policy, let us consider the following problem. A metallic connecting rod is subject to continuous degradation due to repeated tension cycles and crack propagation. It is considered to be 
failed after a degradation index, associated to a normal-470 $y=100$. A large sample of connecting rods are tested in normal operating conditions and the time to failure values are collected. It is seen that the degradation process can reasonably be modeled by a (stationary) gamma process 475 that fitting the failure probability function $F($.$) in (4)$ on the collected sample yields the parameters $v=0.0625$ and $u=8$, in Table 1. A rough description of the random time to failure can be given with a mean value at approximately 200 hours and a standard deviation of 55 445 hours.

In order to visualize the outcome of the sequential policy, a path from the identified stationary gamma degradation process is generated, and the policy is applied in Figure 6. Inspection, preventive and corrective replacement osts are given in Table 1. Two other degradation paths are displayed to illustrate the variability in the degradation process.

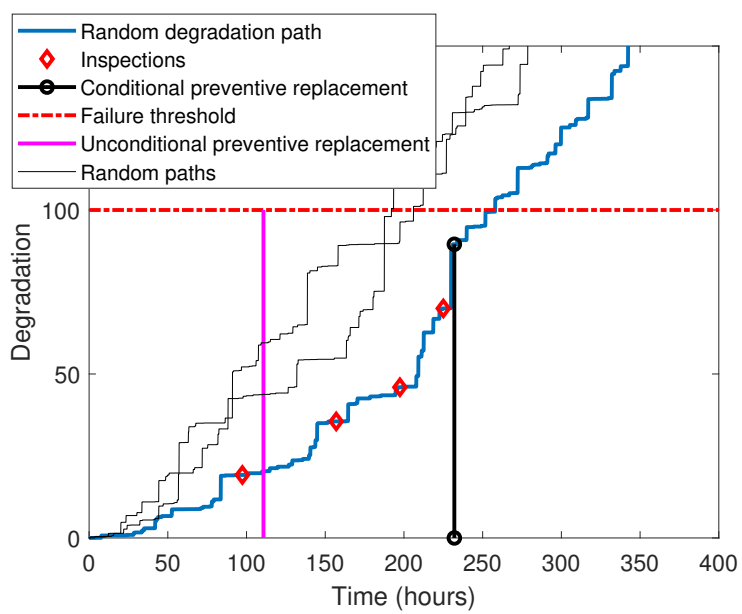

Figure 6: Application of the sequential inspection policy to a random degradation path (thick solid blue line). Other (gamma) degradation paths are also shown (thin solid black lines).

For this example, four inspections have been realized, sequentially, at the best time for inspection. After the fourth inspection, the $\mathrm{VoI}$ is no longer positive for a fifth inspection and the decision is rather to preventively re- ${ }^{490}$ place at the optimal time conditioned on $\left(z_{4}, \tau_{4}\right)$. It is clearly seen that compared to the optimal unconditional preventive replacement time (around $t_{r}=110$ hours), the life of the component is greatly extended with the use of the proposed sequential inspection policy, which leads $t^{495}$ a lower cost per unit time.

\subsection{Experimental validation}

To demonstrate the validity of the proposed sequential inspection policy, the latter is applied on a sample of ${ }^{500}$ degradation paths ( $N=500$ paths) and its average outcome is compared to that of a simple replacement policy or block replacement (BR). The comparison is also made with a more elaborate policy, named Periodic Inspection and Replacement (PIR), see e.g. 17, 18, 12], where inspections are carried out at regular intervals $\Delta T$ and where a crossing of level $M$ at inspection time triggers preventive replacement. The values $\Delta T$ and $M$ are obtained via numerical optimization, with the objective of minimal average cost per unit time, and for the decision context defined in Table 1

The results of the application of all policies on all (500) random paths (as in Figure 6) are given in Figure 7

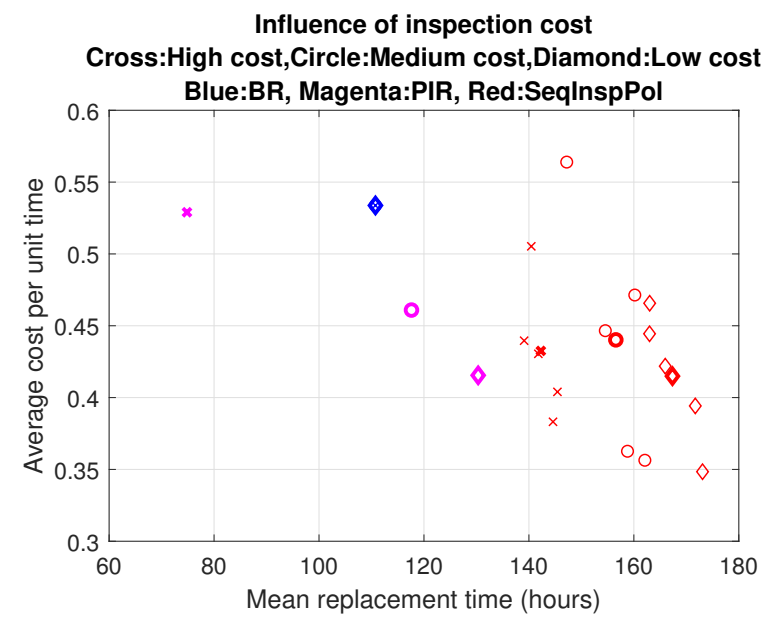

Figure 7: Comparison of the outcomes of the BR, PIR and sequential inspection policy, with varying inspection cost (different markers). For the sequential inspection policy, groups of 100 paths are considered to get an idea on the variability of the result. Larger markers correspond to the average over 500 paths.

It is seen that, no matter the inspection cost, the outcome of the proposed sequential inspection policy is consistently better. Also, with high inspection cost (cross markers), the sequential inspection policy performs much better than PIR. Indeed, when inspections are costly, it is especially relevant to schedule them in an optimal (at least piecewise) manner.

The influence of measurement uncertainty can be visualized in Figure 8, For the latter calculation, at each inspection, a Gaussian measurement error $\epsilon \sim N\left(0, \sigma_{i}\right)$ is added to the true degradation and the obtained sum is the quantity considered as the inspection outcome $z_{i}$ and used for the computation of VoI, which is then based on the averaged conditional cost $C_{\infty \mid z_{i}}^{u}(a)$ as in (25). The true degradation (unknown in principle but used here for validation purposes) is used in order to determine the true failure time, independently of the policy decisions, and compute the outcome of the policy.

It is seen that rather consistently the results are better for the sequential inspection policy. It is also seen that the effect of measurement uncertainty is to somehow shorten the life, as more uncertainty will lead to a more 'careful' policy in order to avoid costly failures. Conditional decisions based on uncertain information happen to be less optimal, thus diminishing the potential VoI.

Additionally, a comparison is proposed between differ- 


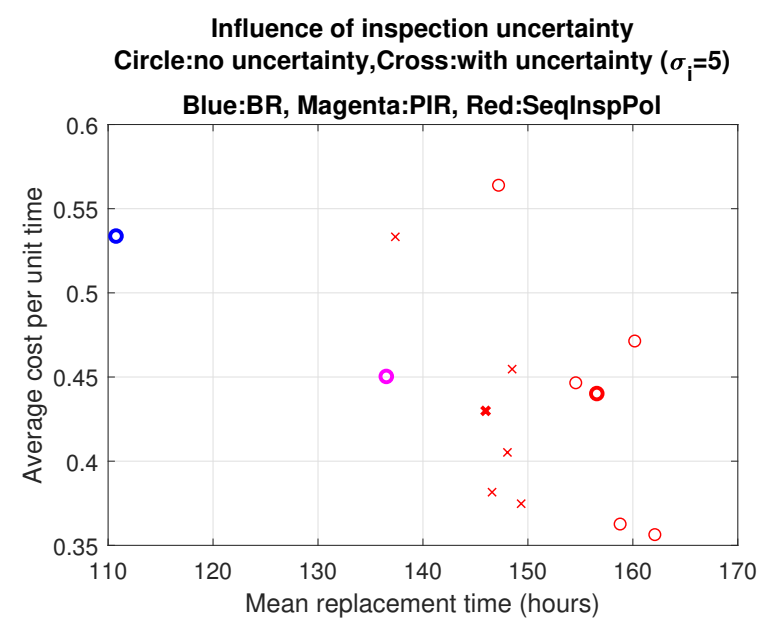

Figure 8: Comparison of the outcome of the BR, PIR and sequential inspection policy, with and without inspection uncertainty (different markers). For the sequential inspection policy, groups of 100 paths $^{525}$ are considered to get an idea on the variability of the result. Larger markers correspond to the average over 500 paths.

ent configurations of the decision context, see Table 1 and Table 2, Such a comparison is visible in Figure 9.

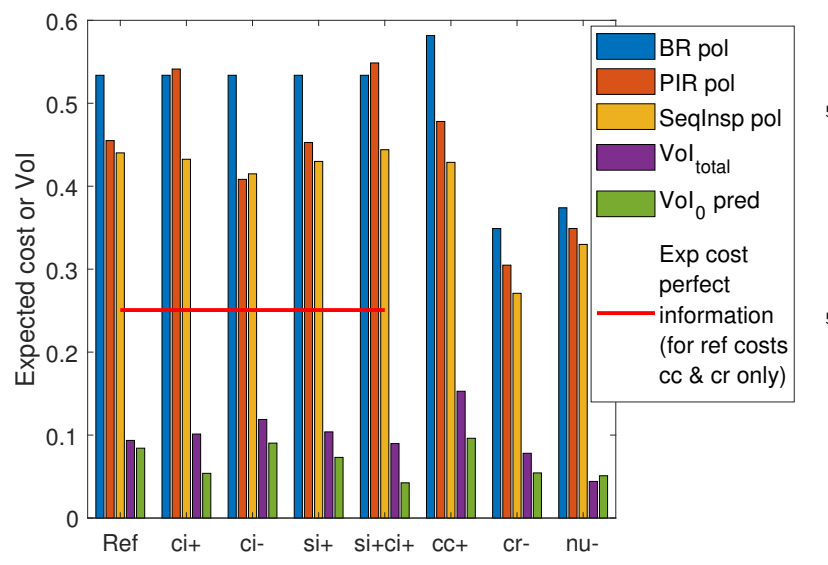

Figure 9: Comparison of BR, PIR and sequential inspection policy outputs, and comparison of the predicted VoI for the first inspection and achieved total VoI, for the different decision contexts. X-axis legend items are described in Table 2

A few remarks can be made. It is seen again that550 with a high inspection cost, the advantage of the sequential inspection policy over PIR is substantial. This advantage tends to disappear with lower inspection cost. The effect of measurement uncertainty is relatively limited here (but variability in the results may be substantial, see Figure 8) .555 Lastly, when the variability of the degradation process is lower, the interest in collecting information decreases.

In Figure 9, the red line, which denotes the achievable outcome with perfect information, is given as an indication. It could represent the theoretical gain associated to a continuous (and free) monitoring system, able to de-560 tect failures just before they happen, therefore triggering

\begin{tabular}{cc} 
Labels & Meaning \\
\hline Ref & Reference case see Table 1 \\
ci + & Inspection cost $c_{i}=5$ istd of $c_{i}=1$ \\
ci- & Inspection cost $c_{i}=0.2$ istd of $c_{i}=1$ \\
si + & Inspection uncertainty $\sigma_{i}=5$ \\
cc + & Corr repl cost $c_{c}=500$ istd of $c_{c}=300$ \\
cr- & Prev repl cost $c_{r}=30$ istd of $c_{r}=50$ \\
nu- & Degradation variability $\nu=2$ istd of $\nu=8$
\end{tabular}

Table 2: Comparison of different policies' outcomes, for different decision contexts. over a multidimensional space of unknown and varying dimension (the number of inspections, not known in advance).

Lastly, it has been noted that, no matter the used pol35 is no consistent difference in terms of the number of failed components in the end. No policy is particularly 'riskier' than another, even if the tendency is to achieve a lower average cost for PIR than for BR and an even lower average cost for the proposed sequential inspection policy. Also, there is no significant difference in the variability of average outcome between PIR and Sequential Inspection Policy (as displayed only for the latter in Figure 7 and 8)

\section{Discussion}

The objective of the work presented in this paper is twofold.

First, it illustrates a concrete implementation of a VoI metric, for a rather simple restriction of the maintenance optimization problem, namely the consideration of only instantaneous and perfect repairs or replacements, of a single component submitted to a stationary gamma degradation process.

Second, it opens the discussion on the assimilation of the notion of $\mathrm{VoI}$ in the field of maintenance optimization or more widely for asset life-cycle management. VoI can be considered as a resource prioritization metric which helps rationalizing data collection or integration, inside the framework of a complex decision-making problem involving uncertainty.

\subsection{Specific remarks on the present case}

In the present case, the VoI metric is used as a guide, along with other simplifications associated to renewal the- 
ory, in the resolution of the sequential decision-making problem of maintenance and inspection planning. Essen-620 tially, it provides guidance on the question: "given stochasic knowledge on the degradation process and maintenance costs, when is it more relevent, if at all, to reduce uncertainty through inspection?".

Let us now consider limits of the present work, theoretical difficulties and potential perspectives.

Unfortunately, for the case of sequential decision making, there is no possible way to compute an optimal policy without approximation or without the consideration of an efficient heuristic (e.g. using VoI). No exact validation can be made as the problem is at least NP-hard, see [40], and global optimality is thus hard to demonstrate. For the aperiodic sequential inspection policy that is proposed in this paper, only piecewise optimality for sequential (yet correlated) problems, is achieved, and it is not straightforward to conclude on the global optimality of the policy ${ }_{635}$ consistently performs better, no matter the decision context.

The prediction of the total expected gain or VoI for the multi-inspection (sequential) policy, that is, a prediction ${ }_{640}$ icy, is hard to compute in advance. The latter would involve mutli-dimensional or conditional integrations, which is computationally difficult.

It seems that the proposed approach is particularly ap- ${ }_{645}$ propriate when inspections are costly, as it would schedule a small number of inspections (sometimes only one or two) with as much gain in expected output as possible. It is more difficult to predict what happens in the case of a large number of cheap inspections, to be distributed on ${ }_{650}$ time domain, and the proposed approach may not be globally optimal in this context. Addressing the questions above, is one perspective to explore.

An interesting problem to consider for sequential decisionmaking and maintenance planing is the possibility to pro- ${ }_{655}$ ess information on future states of the component, i.e. use predictions instead of inspections/estimations. The collected piece of information $z$ would concern not only the current condition but also the future evolution of the degradation process, thus reducing prior uncertainty even ${ }_{66}$ more strongly. To estimate the average outcome associated to decisions conditioned on such information, one would have to propose an adequate conditional expression $C_{\infty \mid z}(a)$ (and the related $p(s \mid z)$ ) and specify $p(z)$, in a manner that is consistent with the prior $p(s)$ used for the computation of the unconditional optimal expected cost. The authors refer to such issue as VoI computation with 'prediction uncertainty', as opposed to 'inspection uncertainty'. This is another perspective to explore.

In theory, an arbitrarily large set of uncertainty reduc- ${ }_{670}$ tion mechanisms $p(s \mid z)$ can be considered.

Lastly, the treatment of problems with more complex decision contexts, e.g. with imperfect repairs or noninstantaneous repairs, requiring semi-renewal or semi-
Markov formulations, which could be interesting from an application perspective, holds less value for the research on VoI from a theoretical standpoint.

\subsection{General and applied remarks on the use of VoI}

More generally, the resource prioritization may not only be needed in the time domain but also in the space domain, e.g. for a distributed structure [41] or in terms of topology, i.e. on different components included in a system structure [34, 42]. Yet, the philosophy of the approach would be the same, as one would try to build a policy that seeks to act, aided by the VoI metric, by collecting information, where or when it is most profitable to do so.

The difficult part of the problem generally lies in the specification of the probability measures of the prior uncertainty $p(s)$ and of the uncertainty reduction mechanism $p(s \mid z)$, as well as the specification of the decision context (here based on $\left.C_{\infty}(a)\right)$. Only when the former are specified can a VoI analysis be implemented using (8). Then, no matter how the problem is formulated, i.e. in time, in space or for a system, the computed VoI necessarily depends on: the decision context, via costs and prior uncertainty, and the uncertainty reduction mechanism. Thus, decisions about information collection will always be problem-dependent. VoI is only a guide, a support metric, for resource prioritization (also a decision problem in itself). For all practical contexts, VoI is a "local and often hand-picked (a particular heuristic), among a continuous set of potential 'observation / uncertaintyreduction mechanisms', loss sensitivity metric within the framework of a so-called exploration-exploitation tradeoff". This, again, explains why the demonstration of optimality is a hard theoretical problem.

A general and quite sensible trend or rule of thumb to keep in mind can be loosely formulated as follows: "the more uncertainty is reduced, in a situation where the average outcome of the conditional optimal action (i.e. the quantity $\left.\min _{a} E_{s}[L(s \mid z, a)]\right)$ is particularly sensitive to the outcome of the collected observation $z$, the more there is a potential for gain, on average, in collecting the considered piece of information".

Lastly, let us note that any 'predicted expected gain', is only as good as the specified stochastic knowledge on uncertainty. Indeed, the prior variability $p(s)$ and the uncertainty reduction mechanism $p(s \mid z)$ are specified for the computation of VoI (so called pre-posterior analysis, see [22]), but they may themselves be imperfect representations of the 'true randomness'.

This is a particularly interesting problem when the reduction of uncertainty is gained through the processing of condition monitoring data. In this latter case, the specification of the precision of the 'observation model', more often seen as 'model validation', is a crucial part for the correct prediction of VoI. Herein lies a potential connection between the questions of optimal decision-making and that of signal and data processing, health estimation, RUL 
(Remaining Useful Life) prediction and more largely PHM

(Prognostic and Health Management), see e.g. [43, 44]. 730

The points made in the last two paragraphs are, in the opinion of the authors, those which deserve the most attention in an applied research perspective.

\section{Conclusion}

In this paper, the concept of Value of Information (VoI) is used as a guide for the piecewise optimal resolution of the complex sequential decision-making problem of maintenance optimization and inspection planning. In practice, a VoI metric is computed based on a stationary gamma process model of degradation, on inspection and preven-740 tive replacement actions, and on the conditional cost per unit time function, defined in the framework of renewal theory. This metric is used for the specification of an aperiodic sequential inspection and condition-based pol-745 icy, which schedules inspections when it is most profitable, in terms of potential gain on average cost, to do so. Inspections times are determined sequentially, in a piecewise optimal manner, until there is no more positive return to ${ }^{750}$ be expected from a new inspection. With experimental validation, it is shown that the latter policy performs consistently better than block replacement or periodic inspection and replacement policies, especially when inspections ${ }^{755}$ are costly.

Through an averaging on the conditional decisions taken lection mechanism, e.g. a noisy inspection or an uncer- ${ }^{76}$ tain state estimation based on the processing of condition monitoring data, this policy can be alimented with imperfect information while retaining its piecewise optimal structure.

On a broader scope, theoretical difficulties and perspectives on the possible use of VoI for the issue of life-cycle management, have been discussed. A VoI analysis implies both a formulation step (defining costs, prior uncertainty ${ }^{770}$

710 and an uncertainty reduction mechanism) and a computation step, where unconditional and conditional expectations and optimizations have to be carried out. Then, for any particular application, $\mathrm{VoI}$ can serve as a resource pri- ${ }^{775}$ oritization metric for time (sequential problems), space or 715 topology(system)-related information collection problems. Different solutions or policies for information collection or uncertainty reduction can be compared. Eventually, ${ }^{780}$ the information collection problem generally constitutes only one part of a larger decision-making problem, where an exploration-exploitation tradeoff is to be reached and where optimality is hard to guarantee. For the resolution ${ }^{785}$ of this larger problem, e.g. maintenance optimization, a VoI metric can be an interesting support.

More precisely, the following perspectives are considered. The question on the global optimality of sequential ${ }^{790}$ (multiple) inspections has to be analyzed more deeply. A study of the consequences of a poor specification of the decision context or of the probability measures necessary for the computation of VoI would also provide interesting information. This is especially true in a practical case where uncertainty reduction is achieved through the processing of condition monitoring data. Also, it would be interesting to consider a formulation for a time-varying problem, in which the information collected relates not only to current condition of the component but also to 'anticipated' or 'predicted' future conditions, thus reducing uncertainty in the time domain even more strongly.

\section{References}

[1] R. E. Barlow, F. Proschan, Mathematical theory of reliability, Vol. 17, Siam, 1967.

[2] M. Abdel-Hameed, A gamma wear process, IEEE transactions on Reliability 24 (2) (1975) 152-153.

[3] P. Thoft-Christensen, J. D. Sorensen, Optimal strategy for inspection and repair of structural systems, Civil Engineering Systems 4 (2) (1987) 94-100.

[4] K. S. Park, Optimal continuous-wear limit replacement under periodic inspections, IEEE Transactions on reliability 37 (1) (1988) 97-102.

[5] Y. Mori, B. R. Ellingwood, Maintaining reliability of concrete structures. ii: optimum inspection/repair, Journal of Structural Engineering 120 (3) (1994) 846-862.

[6] J. M. van Noortwijk, H. E. Klatter, Optimal inspection decisions for the block mats of the eastern-scheldt barrier, Reliability Engineering \& System Safety 65 (3) (1999) 203-211.

[7] R. Dekker, Applications of maintenance optimization models: a review and analysis, Reliability engineering \& system safety 51 (3) (1996) 229-240.

[8] H. Wang, A survey of maintenance policies of deteriorating systems, European journal of operational research 139 (3) (2002) 469-489.

[9] D. M. Frangopol, M.-J. Kallen, J. M. van Noortwijk, Probabilistic models for life-cycle performance of deteriorating structures: review and future directions, Progress in Structural Engineering and Materials 6 (4) (2004) 197-212.

[10] J. M. van Noortwijk, A survey of the application of gamma processes in maintenance, Reliability Engineering \& System Safety 94 (1) (2009) 2-21.

[11] A. H. Christer, W. Wang, A simple condition monitoring model for a direct monitoring process, European journal of operational research 82 (2) (1995) 258-269.

[12] A. Grall, C. Bérenguer, L. Dieulle, A condition-based maintenance policy for stochastically deteriorating systems, Reliability Engineering \& System Safety 76 (2) (2002) 167-180.

[13] A. Grall, L. Dieulle, C. Bérenguer, M. Roussignol, Continuoustime predictive-maintenance scheduling for a deteriorating system, IEEE transactions on reliability 51 (2) (2002) 141-150.

[14] L. Dieulle, C. Bérenguer, A. Grall, M. Roussignol, Sequential condition-based maintenance scheduling for a deteriorating system, European Journal of operational research 150 (2) (2003) 451-461.

[15] M. Crowder, J. Lawless, On a scheme for predictive maintenance, European Journal of Operational Research 176 (3) (2007) 1713-1722.

[16] M. Marseguerra, E. Zio, L. Podofillini, Condition-based maintenance optimization by means of genetic algorithms and monte carlo simulation, Reliability Engineering \& System Safety 77 (2) (2002) 151-165.

[17] K. T. Huynh, A. Barros, C. Berenguer, I. T. Castro, A periodic inspection and replacement policy for systems subject to competing failure modes due to degradation and traumatic events, Reliability Engineering \& System Safety 96 (4) (2011) 497-508.

[18] K. T. Huynh, A. Barros, C. Bérenguer, Maintenance decisionmaking for systems operating under indirect condition monitoring: value of online information and impact of measurement un- 

425 .

[19] S. Madanat, Optimal infrastructure management decisions under uncertainty, Transportation Research Part C: Emerging Technologies 1 (1) (1993) 77-88.

[20] H. Ellis, M. Jiang, R. B. Corotis, Inspection, maintenance, and repair with partial observability, Journal of Infrastructure Systems 1 (2) (1995) 92-99.

[21] K. G. Papakonstantinou, M. Shinozuka, Planning structural inspection and maintenance policies via dynamic programming875 and markov processes. part i: theory, Reliability Engineering \& System Safety 130 (2014) 202-213.

[22] H. Raiffa, Applied statistical decision theory, Div. of Research, Graduate School of Business Administration, Harvard Univ., 1961.

[23] M. H. DeGroot, Uncertainty, information, and sequential experiments, The Annals of Mathematical Statistics 33 (2) (1962) 404-419.

[24] R. A. Howard, Information value theory, IEEE Transactions on systems science and cybernetics 2 (1) (1966) 22-26.

[25] M. Pozzi, A. Der Kiureghian, Assessing the value of information for long-term structural health monitoring, in: Health monitoring of structural and biological systems 2011, Vol. 7984, International Society for Optics and Photonics, 2011, p. 79842W.

[26] D. Straub, Value of information analysis with structural reliability methods, Structural Safety 49 (2014) 75-85.

[27] D. Zonta, B. Glisic, S. Adriaenssens, Value of information: impact of monitoring on decision-making, Structural Control and Health Monitoring 21 (7) (2014) 1043-1056.

[28] K. Konakli, B. Sudret, M. H. Faber, Numerical investigations into the value of information in lifecycle analysis of structural systems, Asce-asme Journal of Risk and Uncertainty in Engineering Systems, Part A: Civil Engineering 2 (3) (2015) B4015007.

[29] D. Straub, E. Chatzi, E. Bismut, W. Courage, M. Döhler, M. H. Faber, J. Köhler, G. Lombaert, P. Omenzetter, M. Pozzi, et al., Value of information: a roadmap to quantifying the benefit of structural health monitoring, in: ICOSSAR - 12thInternational Conference on Structural Safety, 2017.

[30] K. Papakonstantinou, C. Andriotis, H. Gao, E. Chatzi, Quantifying the value of structural monitoring for decision making, in: 13th International Conference on Applications of Statistics and Probability in Civil Engineering(ICASP13), 2019.

[31] M. H. Faber, Risk-based inspection: the framework, Structural engineering international 12 (3) (2002) 186-195.

[32] D. Straub, M. H. Faber, Risk based inspection planning for structural systems, Structural safety 27 (4) (2005) 335-355.

[33] M.-J. Kallen, J. M. van Noortwijk, Optimal maintenance decisions under imperfect inspection, Reliability engineering \& system safety 90 (2-3) (2005) 177-185.

845 [34] M. Memarzadeh, M. Pozzi, Value of information in sequential decision making: component inspection, permanent monitoring and system-level scheduling, Reliability Engineering \& System Safety 154 (2016) 137-151.

[35] M. Memarzadeh, M. Pozzi, Integrated inspection scheduling and maintenance planning for infrastructure systems, Computer-Aided Civil and Infrastructure Engineering 31 (6) (2016) 403-415.

[36] R. Srinivasan, A. K. Parlikad, Value of condition monitoring in infrastructure maintenance, Computers \& Industrial Engineering 66 (2) (2013) 233-241.

[37] A. Daneshkhah, N. G. Stocks, P. Jeffrey, Probabilistic sensitivity analysis of optimised preventive maintenance strategies for deteriorating infrastructure assets, Reliability Engineering \& System Safety 163 (2017) 33-45.

[38] S. Li, M. Pozzi, What makes long-term monitoring convenient? a parametric analysis of value of information in infrastructure maintenance, Structural Control and Health Monitoring 26 (5).

[39] S. M. Ross, Introduction to probability models, Academic press, 2014.

[40] A. Krause, C. Guestrin, Optimal value of information in graph- ical models, Journal of Artificial Intelligence Research 35 (2009) $557-591$

41] C. Malings, M. Pozzi, Value of information for spatially distributed systems: application to sensor placement, Reliability Engineering \& System Safety 154 (2016) 219-233.

[42] W. Fauriat, E. Zio, An importance measure to assess the value of a component inspection policy, in: 3rd International Conference on System Reliability and Safety (ICSRS), IEEE, 2018, pp. 368-375.

[43] A. K. S. Jardine, D. Lin, D. Banjevic, A review on machinery diagnostics and prognostics implementing condition-based maintenance, Mechanical systems and signal processing 20 (7) (2006) 1483-1510.

[44] X.-S. Si, W. Wang, C.-H. Hu, D.-H. Zhou, Remaining useful life estimation-a review on the statistical data driven approaches, European journal of operational research 213 (1) (2011) 1-14. 